Gazi University
Journal of Science
http://dergipark.gov.tr/gujs

\title{
Theoretical Analysis of the Weibull Alpha Power Inverted Exponential Distribution: Properties and Applications
}

\author{
Eferhonore EFE-EYEFIA (D) Joseph Thomas EGHWERIDO* (D) Samuel Chiabom ZELIBE \\ Department of Mathematics and Computer Science, Federal University of Petroleum Resources, Effurun Delta State, Nigeria.
}

Highlights
- The article focuses on a class of inverted exponential distribution.
- The proposed distribution aims at proposing a better flexible model for lifetime data.
- The performance is validated by application of real life data in existing literature.
- Results indicate that the new model competes favourably well with other distributions.

\begin{tabular}{l} 
Article Info \\
\hline \\
Received: 10/03/2019 \\
Accepted: 05/07/2019 \\
Keywords \\
\hline Alpha Power \\
Inverted exponential \\
Life time distribution \\
Maximum likelihood \\
Weibull
\end{tabular}

\begin{abstract}
This article proposed a Weibull-Alpha Power Inverted Exponential (WAPIE) distribution for lifetime processes. Statistical properties of this distribution such as survival, hazard, reversed hazard, cumulative, odd functions, kurtosis, quantiles, skewness, order statistics and the entropies were derived. Parameters of this family of distribution were also obtained by maximum likelihood method. The behaviour of the estimators was studied through simulation. The behavior of the new developed distribution was further examined through real life data. The WAPIE distribution competes favourably well with other distributions.
\end{abstract}

\section{INTRODUCTION}

Lifetime distributions have received several attentions over the years. Thus, its interest has grown over time. Researchers in distribution theory do this either by introducing a new parameter to make the distribution of interest more flexible or possibly produce a new family of distribution [1]. The Weibull distribution was proposed by a famous statistician called Weibull in 1951[2]. This Weibull distribution has a wide range of application in modelling lifetime processes, failure time process, mechanical, electrical system and even in machine learning. [3] proposed the generalized odd Weibull generated family of distributions, [4] developed the generalized exponential distribution and the failure time data were modeled by Lehmann alternatives in [5], Kumaraswamy-inverse exponential distribution was proposed in [6], the properties of the exponentiated generalized inverted exponential distribution was examined in [7], with the transmuted inverse exponential extensively developed in [8], the exponentiated generalized-G Poisson family of distributions was proposed in [9], the generalized transmuted-G family of distributions was proposed in [10], [11] introduced a new family of distributions, [12] proposed the exponentiated generalized class of distributions, [13] proposed the Kumaraswamy Weibull and proposed the McDonald Weibull model in [14], [15] proposed the Burr X generator of distributions and proposed the beta Weibull$\mathrm{G}$ family in [16], The transmuted Topp-Leone $\mathrm{G}$ was proposed in [17], [18] proposed the generalization of the inverse exponential Distribution, with the Lomax distribution in [19] and [20] proposed a class or family of distribution called sum of exponentially distributed random variables. This class of exponential distribution plays important role for a process with continuous memory-less random processes with a constant failure rate which is almost impossible in real life cases. Hence, to account for this disadvantage 
[21] introduced the inverted exponential (IE) distribution with an inverted bathtub failure rate which was further studied and examined by [22-28] proposed the truncated-exponential skewsymmetric distributions and [29] proposed the alpha power Weibull distribution.

The Weibull-G family of distribution was proposed by [30] with a cumulative distribution function given as:

$$
F(t)=\int_{0}^{\frac{G(t)}{1-G(t)}} \varphi \beta t^{\beta-1} e^{-\varphi t^{\beta-1}} d t \quad \varphi, \beta>0 .
$$

Where $\alpha$ and $\beta$ are the shape and scale parameters respectively. The pdf in Equation (1) can be explicitly expressed as

$$
F(t)=1-\exp \left\{-\varphi\left[\frac{G(t)}{1-G(t)}\right]^{\beta}\right\} \quad \varphi, \beta>0 .
$$

The corresponding (pdf) of the Weibull G-family of distribution is given as

$$
f(t)=\varphi \beta g(t) \frac{[G(t)]^{\beta-1}}{[1-G(t)]^{\beta+1}} \exp \left\{-\varphi\left[\frac{G(t)}{1-G(t)}\right]^{\beta}\right\} \quad \varphi, \beta>0 .
$$

The inverted exponential (IE) distribution has an inverted bathtub hazard rate function with the probability density function (pdf) given in [31] as

$$
f_{I E}(t)=\frac{\lambda}{t} \exp \left(-\frac{\lambda}{t}\right) \quad t>0, \quad \lambda>0
$$

where $\lambda$ is the parameter of the $(I E)$. The cumulative distribution function (cdf) is given as

$$
F_{I E}(t)=\exp \left(-\frac{\lambda}{t}\right) \quad t>0, \quad \lambda>0 \text {. }
$$

[31] proposed the alpha power inverted exponential distribution $(A P I E)$ with pdf given as

$$
f_{\text {APIE }}(t)=\frac{\lambda \log \alpha}{t^{2}(\alpha-1)} \exp \left(-\frac{\lambda}{t}\right) \alpha^{\exp \left(-\frac{\lambda}{t}\right)} \quad \text { for } \alpha>0, \quad \alpha \neq 1
$$

The cumulative distribution function of the $(A P I E)$ is given as

$$
F_{A P I E}(t)=\frac{\alpha^{\exp \left(-\frac{\lambda}{t}\right)}-1}{\alpha-1}, \quad \alpha>0, \quad \alpha \neq 1
$$

Motivated by the studies based on the results obtained from the literature research such as the alpha power inverted exponential (APIE), inverted exponential (IE) and the alpha power transmuted (APT) distributions, the Weibull-Alpha Power Inverted Exponential Distribution (WAPIE) four parameters distribution is proposed using Weibull distribution characterizations. Its major characteristic is that two more shape parameters are added to make it more flexible. A comprehensive statistical property of the

WAPIE model is also provided for better view of its applications. This model aims to attract wider range of application in machine learning, medicine, engineering and other related areas.

In this article, the Weibull-Alpha Power Inverted Exponential Distribution (WAPIE) four parameters distribution is developed and proposed as motivated by the alpha power inverted exponential (APIE), 
inverted exponential (IE) and the alpha power transmuted (APT) distributions method using the Weibull characterizations.

\section{THE WEIBULL ALPHA POWER INVERTED EXPONENTIAL DISTRIBUTION}

The Weibull alpha power inverted exponential distribution is a member of the alpha power exponential distribution with the following probability density function

$$
\begin{aligned}
& f_{\text {WAPIE }}(x)=\varphi \beta \frac{\lambda \log \alpha}{x^{2}(\alpha-1)} \exp \left(-\frac{\lambda}{x}\right) \alpha^{\exp \left(-\frac{\lambda}{x}\right)} \cdot \frac{\left[\frac{\alpha^{\exp \left(-\frac{\lambda}{x}\right)}-1}{\alpha-1}\right]^{\beta-1}}{\left[\frac{\alpha-\alpha^{\exp \left(-\frac{\lambda}{x}\right)}}{\alpha-1}\right]^{\beta+1}} \exp \left\{-\varphi\left[\frac{\alpha^{\exp \left(-\frac{\lambda}{x}\right)}-1}{\alpha-\alpha^{\exp \left(-\frac{\lambda}{x}\right)}}\right]^{\beta}\right\} \\
& \varphi, \alpha, \beta, \lambda>0 ; \alpha \neq 1 .
\end{aligned}
$$

The associated cumulative distribution function is given as

$$
F_{\text {WAPIE }}(x)=1-\exp \left(-\varphi\left(\frac{\alpha^{\exp \left(-\frac{\lambda}{x}\right)}-1}{\alpha-\alpha^{\exp \left(-\frac{\lambda}{x}\right)}}\right)^{\beta}\right) \quad \varphi, \alpha, \beta, \lambda>0 ; \quad \alpha \neq 1 .
$$

Figure 1 is the plot of the pdf of the WAPIE distribution for different values of parameters.

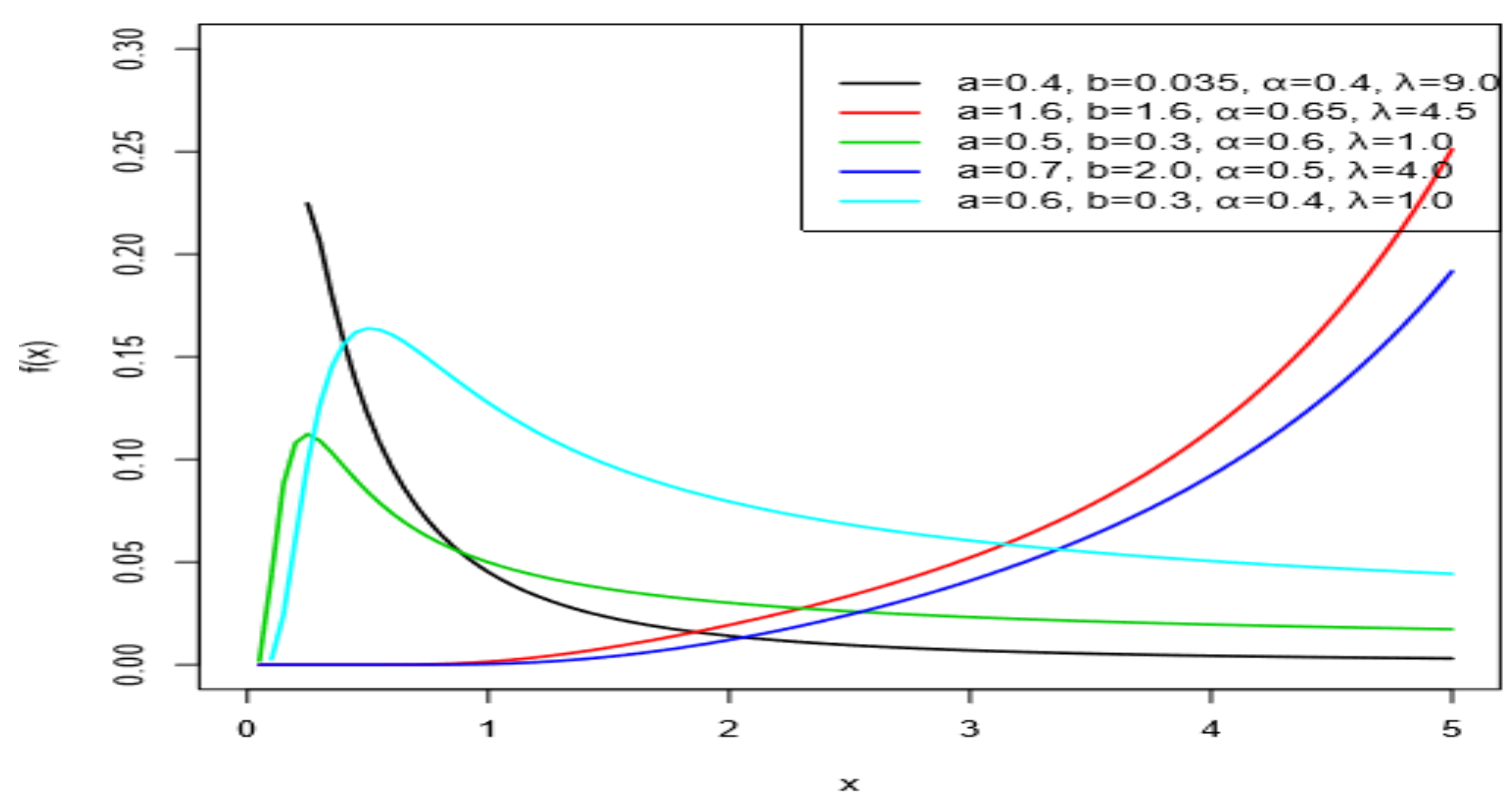

Figure 1. The pdf of the WAPIE distribution with different parameter values 


\section{Remark 1.}

The shape of the pdf of the WAPIE distribution could be bathtub or skewed depending on the values of the parameters.

Figure 2 is the plot of the cdf of the WAPIE distribution for different values of parameters. The shape of the cdf of the WAPIE distribution could be bathtub depending on the values of the parameters

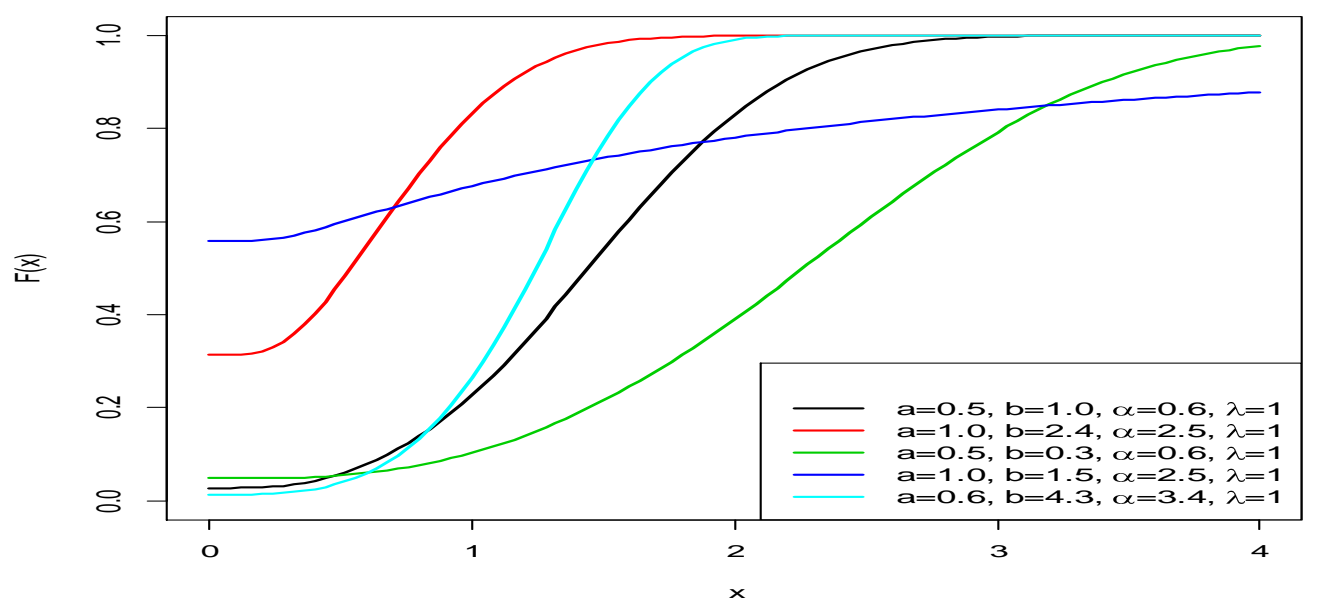

Figure 2. The cdf of the WAPIE distribution with different parameters

\section{Remark 2.}

The shape of the cdf of the WAPIE distribution is increasing depending on the values of the parameters.

\subsection{Parameter Estimation for the Weibull Alpha Power Inverted Exponential Distribution Formulation}

Let $x_{1}, x_{2}, \cdots, x_{n}$ be random variable obtained from a population with weilbull alpha power inverted exponential distribution. Then, the log-likelihood of weilbull alpha power inverted exponential distribution for vector $\Theta=(\varphi, \beta, \alpha, \lambda)^{T}$ can be represented as $\ell_{n}\left(x_{i}, \Theta\right)$ as

$$
\begin{aligned}
& \ell_{n}\left(x_{i}, \Theta\right)=n \log \varphi+n \log \beta+\sum_{i=1}^{n} \log g\left(x_{i}\right)+(\beta-1) \sum_{i=1}^{n} \log G\left(x_{i}\right)-(\beta+1) \sum_{i=1}^{n} \log \left(1-G\left(x_{i}\right)\right) \\
& \quad-\varphi \sum_{i=1}^{n}\left(\frac{G\left(x_{i}\right)}{1-G\left(x_{i}\right)}\right)^{\beta} \cdot \\
& \text { Let } p=\frac{\partial \sum_{i=1}^{n}\left(\frac{G\left(x_{i}\right)}{1-G\left(x_{i}\right)}\right)^{\beta}}{\partial \beta}, r_{i}=\frac{\partial \sum_{i=1}^{n} \log g\left(x_{i}\right)}{\partial \alpha}, z_{i}=\frac{\partial \sum_{i=1}^{n} \log G\left(x_{i}\right)}{\partial \alpha}, k_{i}=\frac{\partial \sum_{i=1}^{n} \log \left(1-G\left(x_{i}\right)\right)}{\partial \alpha}, \\
& w_{i}=\frac{\partial \sum_{i=1}^{n}\left(\frac{G\left(x_{i}\right)}{1-G\left(x_{i}\right)}\right)^{\beta}}{\partial \alpha}, t_{i}=\frac{\partial \sum_{i=1}^{n}\left(\frac{G\left(x_{i}\right)}{1-G\left(x_{i}\right)}\right)^{\beta}}{\partial \lambda}, m_{i}=\frac{\partial \sum_{i=1}^{n} \log g\left(x_{i}\right)}{\partial \lambda}, s_{i}=\frac{\partial \sum_{i=1}^{n} \log G\left(x_{i}\right)}{\partial \lambda}, \\
& q_{i}=\frac{\partial \sum_{i=1}^{n} \log \left(1-G\left(x_{i}\right)\right)}{\partial \lambda} .
\end{aligned}
$$


Taking partial derivative of Equation (10) with respect to the parameters, we have

$$
\begin{aligned}
& U_{\varphi}=\frac{n}{\varphi}-\sum_{i=1}^{n}\left(\frac{G\left(x_{i}\right)}{1-G\left(x_{i}\right)}\right)^{\beta} \\
& U_{\beta}=\frac{n}{\beta}+\sum_{i=1}^{n} \log G\left(x_{i}\right)-\sum_{i=1}^{n} \log \left(1-G\left(x_{i}\right)\right)-\varphi p \\
& U_{\alpha}=r_{i}+(\beta-1) z_{i}-(\beta+1) k_{i}-\varphi w_{i} . \\
& U_{i}=m_{i}+(\beta-1) s_{i}-(\beta+1) q_{i}-\varphi t_{i} .
\end{aligned}
$$

Thus, setting $U_{\alpha}=U_{\beta}=U_{\lambda}=U_{\alpha}=0$. The solution to the nonlinear equation for the parameters can be obtained using $\mathrm{R}$ software, MATLAB, and MAPLE. Thus, yield the maximum likelihood estimate $\hat{\Theta}=(\hat{\alpha}, \hat{\beta}, \hat{\lambda}, \hat{\alpha})$.

\section{SOME STATISTICAL PROPERTIES OF THE WEIBULL ALPHA POWER INVERTED EXPONENTIAL DISTRIBUTION FORMULATION}

In this section, we study some statistical properties of the WAPIE distribution. It comprise reliability analysis, hazard rate function, cumulative hazard rate function, reserved hazard function, odds function, quantile function, moments, and order statistics.

\subsection{Reliability Analysis}

The survival function of the WAPIE distribution for variable $X>0$ is given as

$$
S_{\text {WAPIE }}(x)=\exp \left(-\varphi\left(\frac{\alpha^{\exp \left(-\frac{\lambda}{x}\right)}-1}{\alpha-\alpha^{\exp \left(-\frac{\lambda}{x}\right)}}\right)^{\beta}\right), \quad \text { for } \varphi>0, \alpha \neq 1, \beta, \lambda>0 \text {. }
$$

\subsection{Hazard Rate Function}

The Hazard rate function of the WAPIE is given as

$$
H_{\text {WAPIE }}(x)=\varphi \beta \frac{\lambda \log \alpha}{x^{2}(\alpha-1)} \exp \left(-\frac{\lambda}{x}\right) \alpha^{\exp \left(-\frac{\lambda}{x}\right)}\left(\frac{\left(\frac{\alpha^{\exp \left(-\frac{\lambda}{x}\right)}-1}{\alpha-1}\right)^{\beta-1}}{\left(\frac{\alpha-\alpha^{\exp \left(-\frac{\lambda}{x}\right)}}{\alpha-1}\right)^{\beta+1}}\right), \quad \varphi, \beta, \lambda>0, \quad \alpha \neq 1 .
$$

\subsection{Cumulative Hazard rate Function}

The cumulative hazard function $(\mathrm{CH})$ of the WAPIE distribution is given as

$$
C H_{\text {WAPIE }}(x)=\varphi\left(\frac{\alpha^{\exp \left(-\frac{\lambda}{x}\right)}-1}{\alpha-\alpha^{\exp \left(-\frac{\lambda}{x}\right)}}\right)^{\beta}, \quad \varphi, \beta, \lambda>0, \alpha \neq 1 \text {. }
$$

\subsection{Reversed Hazard Function}

The WAPIE distribution has a reversed hazard function rate (RH) given as 


$$
=\frac{\varphi \beta \lambda \log \alpha \exp \left(-\frac{\lambda}{x}\right) \alpha^{\exp \left(-\frac{\lambda}{x}\right)}\left(\frac{\alpha^{\exp \left(-\frac{\lambda}{x}\right)}-1}{\alpha-1}\right)^{\beta-1} \exp \left(-\varphi\left(\frac{\alpha^{\exp \left(-\frac{\lambda}{x}\right)}-1}{\left.\left.\alpha-\alpha^{\exp \left(-\frac{\lambda}{x}\right)}\right)^{\beta}\right)},\right.\right.}{x^{2}(\alpha-1)\left(\frac{\alpha-\alpha^{\exp \left(-\frac{\lambda}{x}\right)}}{\alpha-1}\right)^{\beta+1}\left(1-\exp \left(-\varphi\left(\frac{\alpha^{\exp \left(-\frac{\lambda}{x}\right)}-1}{\left.\alpha-\alpha^{\exp \left(-\frac{\lambda}{x}\right)}\right)}\right)\right)\right.}
$$

$\varphi, \beta, \lambda>0, \alpha \neq 1$.

\subsection{Odds Function}

The odds function $(\mathrm{O})$ of the WAPIE distribution is given as

$$
O_{\text {WAPIE }}(x)=\exp \left(\varphi\left(\frac{1-\alpha^{\exp \left(-\frac{\lambda}{x}\right)}}{\alpha-\alpha^{\exp \left(-\frac{\lambda}{x}\right)}}\right)^{\beta}\right)-1 \quad \varphi, \beta, \lambda>0, \alpha \neq 1
$$

\subsection{Quantile and Median Function}

The Quantile function of the WAPIE distribution is derived from the equation

$Q(u)=F^{-1}(x)$.

Therefore, the quantile function of the Weibull-Alpha power inverted exponential distribution is given as $Q(u)=-\lambda\left[\log \left[(\log \alpha)^{-1} \log \left((\alpha-1)\left(\left(1+\left(-\varphi^{-1} \log (1-u)\right)^{\frac{1}{\beta}}\right)^{-1}\left(-\varphi^{-1} \log (1-u)\right)\right)+1\right)\right]\right]^{-1}$

where $\mathrm{u} \sim$ uniform $[0,1]$.

Using the WAPIE distribution, random numbers $X$ generated from the WAPIE distribution is given by $x=-\lambda\left[\log \left[(\log \alpha)^{-1} \log \left((\alpha-1)\left(\left(1+\left(-\varphi^{-1} \log (1-u)\right)^{\frac{1}{\beta}}\right)^{-1}\left(-\varphi^{-1} \log (1-u)\right)\right)+1\right)\right]\right]^{-1}$.

The median of the WAPIE distribution can be obtained by substituting $u=\frac{1}{2}$ in Equation (22) as Median $=-\lambda\left[\log \left[(\log \alpha)^{-1} \log \left((\alpha-1)\left(\left(1+\left(-\varphi^{-1} \log \left(\frac{1}{2}\right)\right)^{\frac{1}{\beta}}\right)^{-1}\left(-\varphi^{-1} \log \left(\frac{1}{2}\right)\right)\right)+1\right)\right]\right]^{-1}$

Then, the $25^{\text {th }}$ percentile and the $75^{\text {th }}$ percentile are given by Equations (24) and (25) respectively 


$$
\begin{aligned}
& Q_{1}=-\lambda\left[\log \left[(\log \alpha)^{-1} \log \left((\alpha-1)\left(\left(1+\left(-\varphi^{-1} \log (0.75)\right)^{\frac{1}{\beta}}\right)^{-1}\left(-\varphi^{-1} \log (0.75)\right)\right)+1\right)\right]\right]^{-1} \\
& Q_{3}=-\lambda\left[\log \left[(\log \alpha)^{-1} \log \left((\alpha-1)\left(\left(1+\left(-\varphi^{-1} \log (0.25)\right)^{\frac{1}{\beta}}\right)^{-1}\left(-\varphi^{-1} \log (0.25)\right)\right)+1\right)\right]\right]^{-1} .
\end{aligned}
$$

The Bowley's formula for finding the coefficient of skewness is given as

$$
S_{k}(B)=\frac{x_{0.75}-2 x_{0.5}+x_{0.25}}{x_{0.75}-x_{0.25}} \text {. }
$$

The Moor's formula for coefficient of kurtosis is given as

$$
K_{k}(u)=\frac{x_{0.875}-x_{0.625}-x_{0.375}+x_{0.125}}{x_{0.75}-x_{0.25}} \text {. }
$$

\subsection{The $r^{\text {th }}$ Moments}

The $r^{\text {th }}$ moment of the WAPIE distribution is given as

$$
\begin{aligned}
& x^{r} \varphi \beta \frac{\lambda \log \alpha}{x^{2}(\alpha-1)} \exp \left(-\frac{\lambda}{x}\right) \alpha^{\exp \left(-\frac{\lambda}{x}\right)} \cdot \frac{\left[\frac{\alpha^{\exp \left(-\frac{\lambda}{x}\right)}-1}{\alpha-1}\right]^{\beta-1}}{\left[\frac{\alpha-\alpha^{\exp \left(-\frac{\lambda}{x}\right)}}{\alpha-1}\right]^{\beta+1}} \\
& \times \exp \left\{-\varphi\left[\frac{\alpha^{\exp \left(-\frac{\lambda}{x}\right)}-1}{\alpha-\alpha^{\exp \left(-\frac{\lambda}{x}\right)}}\right]^{\beta}\right\} d x .
\end{aligned}
$$

\subsection{The Probability Weighted Moments}

This is a class of moments used to derived estimators of the quantiles and parameters of the WAPIE distribution expressed in inverse form. Thus, for a random variable $X$, the probability weighted moments denoted by

$$
\tau_{r, s}=E\left(X^{r} F_{\text {WAPIE }}(x)^{s}\right)=\int_{-\infty}^{\infty} x^{r} f_{\text {WAPIE }}(x) F_{\text {WAPIE }}(x)^{s} d x
$$

\subsection{Distribution of Order Statistics}

Suppose $x_{1}, x_{2}, \cdots, x_{n}$ be a random sample drawn from an infinite population with a pdf of the WAPIE distribution. Then the pdf of the $k^{\text {th }}$ order statistics is given by 


$$
\begin{aligned}
& g_{k}(x)=\frac{n !}{(k-1) !(n-k) !}\left[1-\exp \left(-\varphi\left(\frac{\alpha^{\exp \left(-\frac{\lambda}{x}\right)}-1}{\left.\left.\alpha-\alpha^{\exp \left(-\frac{\lambda}{x}\right)}\right)^{\beta}\right)}\right]^{k-1}\left[\operatorname { e x p } \left(-\varphi\left(\frac{\alpha^{\exp \left(-\frac{\lambda}{x}\right)}-1}{\left.\alpha-\alpha^{\exp \left(-\frac{\lambda}{x}\right)}\right)^{\beta}}\right]^{n-k-1}\right.\right.\right.\right. \\
& \times \varphi \beta \frac{\lambda \log \alpha}{x^{2}(\alpha-1)} \exp \left(-\frac{\lambda}{x}\right) \alpha^{\exp \left(-\frac{\lambda}{x}\right)} \cdot \frac{\left[\frac{\alpha^{\exp \left(-\frac{\lambda}{x}\right)}-1}{\alpha-1}\right]^{\beta-1}}{\left[\frac{\alpha-\alpha^{\exp \left(-\frac{\lambda}{x}\right)}}{\alpha-1}\right]^{\beta+1}} .
\end{aligned}
$$

We obtain the minimum and maximum order statistics when $k=1$ and $k=n$ respectively.

\section{SIMULATION STUDY}

A simulation is carried out to test the efficiency of the weibull alpha power inverted exponential distribution. Table 1 shows the simulation for different values of parameters for skewness, kurtosis, median, 25 th and $75^{\text {th }}$ percent of the WAPIE distribution. Increase parameters decreases the skewness and kurtosis but increases the percentile

\begin{tabular}{|c|c|c|c|c|c|c|c|c|}
\hline \multicolumn{4}{|c|}{ Parameters } & \multirow{2}{*}{ Skewness } & \multirow{2}{*}{ Kurtosis } & \multirow{2}{*}{ Median } & \multirow{2}{*}{$25^{\text {th }}$ percent } & \multirow{2}{*}{$75^{\text {th }}$ percent } \\
\hline$\varphi$ & $\beta$ & $\lambda$ & $\alpha$ & & & & & \\
\hline 0.500 & 0.500 & 0.500 & 2.000 & 0.5560 & 1.6147 & 1.5834 & 0.4393 & 5.5928 \\
\hline 1.000 & 0.500 & 0.500 & 3.000 & 0.5160 & 1.4852 & 0.6355 & 0.2446 & 1.8598 \\
\hline 0.500 & 1.000 & 1.000 & 7.000 & 0.2490 & 0.5722 & 3.8509 & 1.9345 & 7.0381 \\
\hline 1.000 & 1.000 & 1.000 & 20.00 & 0.2305 & 0.5247 & 3.1113 & 1.6891 & 5.3854 \\
\hline 2.000 & 2.000 & 2.000 & 50.00 & 0.0554 & 0.1163 & 7.1088 & 5.2480 & 9.1882 \\
\hline 1.500 & 1.500 & 3.000 & 2.000 & 0.1186 & 0.2539 & 3.8471 & 2.6432 & 5.3750 \\
\hline 2.000 & 1.500 & 1.500 & 3.000 & 0.1153 & 0.2465 & 1.9412 & 1.3385 & 2.7008 \\
\hline 2.500 & 2.000 & 1.500 & 7.000 & 0.0570 & 0.1200 & 2.7222 & 2.0146 & 3.5154 \\
\hline 2.500 & 2.500 & 2.000 & 20.00 & 0.0219 & 0.0473 & 5.5825 & 4.3502 & 6.8701 \\
\hline 3.000 & 3.000 & 3.000 & 50.00 & -0.0017 & $-5.78 \mathrm{e}-05$ & 10.987 & 8.9361 & 13.030 \\
\hline 1.500 & 1.500 & 5.000 & 2.000 & 0.1186 & 0.2540 & 6.4118 & 4.4054 & 8.9583 \\
\hline 2.000 & 1.500 & 5.000 & 3.000 & 0.1153 & 0.2465 & 6.4705 & 4.4618 & 9.0027 \\
\hline 2.500 & 2.000 & 5.000 & 7.000 & 0.0570 & 0.1200 & 9.0740 & 6.7151 & 11.718 \\
\hline 2.500 & 2.500 & 5.000 & 20.00 & 0.0219 & 0.0473 & 13.956 & 10.875 & 17.175 \\
\hline 3.000 & 3.000 & 5.000 & 50.00 & -0.0017 & $-5.78 \mathrm{e}-05$ & 18.311 & 14.894 & 21.717 \\
\hline 1.500 & 1.500 & 7.000 & 2.000 & 0.1186 & 0.2540 & 8.9764 & 6.1676 & 12.542 \\
\hline 2.000 & 1.500 & 7.000 & 3.000 & 0.1153 & 0.2465 & 9.0587 & 6.2465 & 12.604 \\
\hline 2.500 & 2.000 & 7.000 & 7.000 & 0.0570 & 0.1200 & 12.704 & 9.4013 & 16.405 \\
\hline 2.500 & 2.500 & 7.000 & 20.00 & 0.0219 & 0.0473 & 19.537 & 15.226 & 24.045 \\
\hline 3.000 & 3.000 & 7.000 & 50.00 & -0.0017 & $-5.78 \mathrm{e}-05$ & 25.636 & 20.851 & 30.404 \\
\hline
\end{tabular}

Table 1. A simulation study of the weibull alpha power inverted exponential distribution

\section{APPLICATION}

To examine the performance of the WAPIE model with other competing distributions the gas fiber and carbon data real-life datasets were used. We considered the Akaike Information Criteria (AIC), Consistent Akaike Information Criteria (CAIC), Bayesian Information Criteria (BIC), Hannan-Quinn Information 
Criteria (HQIC), The Anderson Darling (A) statistic, Cramer-von Mises statistic (W), Kolmogorov Smirnov (KS) statistic, Log-likelihood and the P value to compare the fits of the WAPIE model to other competing models Kumaraswamy Lomax (KULOMAX), Kumaraswamy Exponential (KUEX), Kumaraswamy-Alpha Power Inverted Exponential (KAPIE), Kumaraswamy Burx11 (KUBUXI), Kumaraswamy Inverse Exponential (KUIEX), Alpha Power Inverted Exponential (APIE) and Exponential (EX)distributions.

\subsection{First Set of Glass Fiber Data}

Datasets were collected for $1.5 \mathrm{~cm}$ strengths of glass fibres data at the UK National Physical Laboratory and was used to test the performance of the WAPIE distribution as used by [30, 32-36]. The observations are as follows:

$0.55,0.74,0.77,0.81,0.84,1.24,0.93,1.04,1.11,1.13,1.30,1.25,1.27,1.28,1.29,1.48,1.36,1.39,1.42$, $1.48,1.51,1.49,1.49,1.50,1.50,1.55,1.52,1.53,1.54,1.55,1.61,1.58,1.59,1.60,1.61,1.63,1.61,1.61$, $1.62,1.62,1.67,1.64,1.66,1.66,1.66,1.70,1.68,1.68,1.69,1.70,1.78,1.73,1.76,1.76,1.77,1.89,1.81$, $1.82,1.84,1.84,2.00,2.01,2.24$

The performance of the WAPIE distribution compared to other distribution is shown in Table 2 and 3.

Table 2. The goodness of fit statistics for the glass fiber's data

\begin{tabular}{|c|c|c|c|c|c|c|}
\hline MODEL & AIC & CAIC & BIC & HQIC & A & W \\
\hline WAPIE & 39.55332 & 40.24298 & 48.12586 & 42.92494 & 0.2668984 & 1.461937 \\
\hline KULOMAX & 43.67962 & 44.36927 & 52.25216 & 47.05124 & 0.3530559 & 1.933592 \\
\hline KUEX & 40.89735 & 41.30413 & 47.32676 & 43.42607 & 0.3352229 & 1.835432 \\
\hline KAPIE & 52.71052 & 53.40017 & 61.28306 & 56.08214 & 0.5063777 & 2.770684 \\
\hline KUBUXI & 47.19761 & 47.88727 & 55.77015 & 50.56923 & 0.4179052 & 2.289465 \\
\hline KUIEX & 50.12111 & 50.52789 & 56.55051 & 52.64983 & 0.4813895 & 2.632409 \\
\hline APIE & 196.3253 & 196.5253 & 200.6116 & 198.0111 & 0.777503 & 4.238456 \\
\hline
\end{tabular}

Table 3. MLEs of parameters WAPIE distribution for the glass fibers

\begin{tabular}{|c|l|l|l|l|}
\hline PARAMETERS & VALUES & St.D & Inf. 95\% CI & Sup. 95\% CI \\
\hline$\varphi$ & 0.005860867 & 0.003039024 & $-9.551092 \mathrm{e}-05$ & 0.01181724 \\
\hline$\beta$ & 4.979656013 & 0.484188807 & $4.030663 \mathrm{e}+00$ & 5.92864864 \\
\hline$\lambda$ & 0.365468737 & 0.140473092 & $9.014654 \mathrm{e}-02$ & 0.64079094 \\
\hline$\alpha$ & 2.035789436 & 1.976538211 & $-1.83815 \mathrm{e}+00$ & 5.90973314 \\
\hline
\end{tabular}

From the result it shows that the WAPIE distribution has the smallest AIC, CAIC, BIC, HQIC, A and W when compared to KAPIE, KUBUXI, KULOMAX, KUEX, KUIEX and APIE Distribution. The measure of the test statistics is shown in Table 4 below.

Table 4. Measure of test statistics collections for the glass fibers [37]

\begin{tabular}{|c|c|c|c|}
\hline TEST STATISTICS & KS Statistic & KS p-value & log-likelihood \\
\hline WAPIE & 0.1646002 & 0.06583302 & 15.77666 \\
\hline KAPIE & 0.2100631 & 0.007698024 & 22.35526 \\
\hline KUBUXI & 0.2017095 & 0.01187427 & 19.59881 \\
\hline KULOMAX & 0.1818623 & 0.03098763 & 17.83981 \\
\hline KUEX & 0.1769054 & 0.03877044 & 17.44868 \\
\hline KUIEX & 0.1992272 & 0.0134607 & 22.06056 \\
\hline APIE & 0.4645605 & $3.099632 \mathrm{e}-12$ & 96.16265 \\
\hline
\end{tabular}




\subsection{Second Set of Carbon Data}

Our second set of data is from [37]. It consists of 100 observations taken on breaking stress of carbon fibers (in Gba). The dataset are as follow:

3.7, 2.74, 2.73, 2.5, 3.6, 3.11, 3.27, 2.87, 1.47, 3.11, 4.42, 2.41, 3.19, 3.22, 1.69, 3.28, 3.09, 1.87, 3.15, 4.9, $3.75,2.43,2.95,2.97,3.39,2.96,2.53,2.67,2.93,3.22,3.39,2.81,4.2,3.33,2.55,3.31,3.31,2.85,2.56$, $3.56,3.15,2.35,2.55,2.59,2.38,2.81,2.77,2.17,2.83,1.92,1.41,3.68,2.97,1.36,0.98,2.76,4.91,3.68$, $1.84,1.59,3.19,1.57,0.81,5.56,1.73,1.59,2,1.22,1.12,1.71,2.17,1.17,5.08,2.48,1.18,3.51,2.17$, $1.69,1.25,4.38,1.84,0.39,3.68,2.48,0.85,1.61,2.79,4.7,2.03,1.8,1.57,1.08,2.03,1.61,2.12,1.89$, $2.88,2.82,2.05,3.65$

Tables 5 and 6 show the goodness-of-fit and the performance rating of the WAPIE distribution using several test statistics for the carbon fibers dataset.

Table 5. The goodness of fit statistics for the carbon data

\begin{tabular}{|c|l|l|l|l|l|l|}
\hline MODEL & AIC & CAIC & BIC & HQIC & A & W \\
\hline WAPIE & 290.8659 & 291.287 & 301.2866 & 295.0834 & 0.07010233 & 0.4242744 \\
\hline KULOMAX & 290.9681 & 291.3891 & 301.3888 & 295.1855 & 0.0842262 & 0.4531522 \\
\hline KUEX & 288.7576 & 289.0076 & 296.5731 & 291.9207 & 0.07768531 & 0.4303957 \\
\hline KAPIE & 294.6224 & 295.0435 & 305.0431 & 298.8398 & 0.1338752 & 0.6933353 \\
\hline KUBUXI & 292.8652 & 293.2863 & 303.2859 & 297.0827 & 0.1183604 & 0.6086712 \\
\hline KUIEX & 308.4821 & 308.7321 & 316.2976 & 311.6452 & 0.3207067 & 1.745378 \\
\hline APIE & 422.3312 & 422.455 & 427.5416 & 424.44 & 0.37259 & 2.042718 \\
\hline
\end{tabular}

Table 6. MLEs of parameters WAPIE distribution for the carbon data

\begin{tabular}{|c|l|l|l|l|}
\hline PARAMETERS & VALUES & St.D & Inf. 95\% CI & Sup. 95\% CI \\
\hline$\varphi$ & 0.03428428 & 0.08370638 & -0.1297772 & 0.1983458 \\
\hline$\beta$ & 2.53280436 & 0.25368799 & 2.0355850 & 3.0300237 \\
\hline$\lambda$ & 0.39871544 & 0.57995511 & -0.7379757 & 1.5354066 \\
\hline$\alpha$ & 3.61479397 & 14.43971884 & -24.6865349 & 31.9161229 \\
\hline
\end{tabular}

The measure of the test statistics is shown in Table 7 below.

Table 7. Measure of test statistics collections for the carbon data

\begin{tabular}{|c|l|l|l|}
\hline TEST STATISTICS & KS Statistic & KS p-value & Log-likelihood \\
\hline WAPIE & 0.06283299 & 0.8247003 & 141.433 \\
\hline KAPIE & 0.09093334 & 0.3799776 & 143.3112 \\
\hline KUBUXI & 0.08533937 & 0.4601788 & 142.4326 \\
\hline KULOMAX & 0.07543761 & 0.6198049 & 141.484 \\
\hline KUEX & 0.07046968 & 0.7034028 & 141.3788 \\
\hline KUIEX & 0.130143 & 0.06758742 & 151.241 \\
\hline APIE & 0.3503104 & $4.384659 \mathrm{e}-11$ & 209.1656 \\
\hline
\end{tabular}

\section{CONCLUSION}

The concept of the WAPIE distribution has been well defined and studied. The mathematical expression for the probability density function (pdf) and cumulative distribution function (cdf) were examined. We also derived some of the statistical properties of the WAPIE distribution including survival function, hazard 
function, reversed hazard function, odds function, order statistics, cumulative hazard rate function, quantile and median function. Then the parameter estimation was obtained using the maximum likelihood estimation (MLE) approach. When applied to data the WAPIE distribution better than the KAPIE, KUBUXI, KUEX, KULOMAX, KUIEX, and APIE distributions.

\section{CONFLICTS OF INTEREST}

No conflict of interest was declared by the authors

\section{REFERENCES}

[1] Anake, T. A., Oguntunde, P. E., Odetunmibi, O. A. "On a fractional beta- distribution", International Journal of Mathematics and Computations, 26(1): 26-34, (2015).

[2] Weibull, W. "A statistical distribution function of wide applicability", Journal of Applied Mechanics, Transactions, 18: 293-297, (1951).

[3] Korkmaz, M., Alizadeh M., Yousof, H. M., Butt, N. S. "The generalized odd weibull generated family of distributions: statistical properties and applications", Pakistan Journal of Statistics and Operation Research, 3: 541- 556, (2018).

[4] Gupta, R. D., Kundu, D. “Generalized exponential distribution”, Australian and New Zealand Journal of Statistics, 41 (2): 173-188,(1999).

[5] Gupta, R. C, Gupta, P. I. \& Gupta, R. D. "Modeling failure time data by Lehmann alternatives", Communications in Statistics-Theory and Methods, 27: 887-904, (1998).

[6] Oguntunde, P.E., Babatunde, O.S., Ogunmola, A.O., "Theoretical analysis of the kumaraswamyinverse exponential distribution", International Journal of Statistics and Applications, 4(2): 113-116, (2014a).

[7] Oguntunde, P.E., Adejumo, A., Balogun, O.S. "Statistical properties of the exponentiated generalized inverted exponential distribution", Applied Mathematics, 4(2): 47-55, (2014b).

[8] Oguntunde, P., Adejumo, O. “The transmuted inverse exponential distribution”, International Journal of Advanced Statistics and Probability, 3(1): 1-7 (2014c).

[9] Aryal, G. R., Yousof, H. M. "The exponentiated generalized-G Poisson family of distributions", Economic Quality Control, 32(1): 1-17, (2017).

[10] Nofal, Z. M., Affy, A. Z., Yousof, H. M., Cordeiro, G. M. "The generalized transmuted-G family of distributions", Communications in Statistics Theory and Methods, 46: 4119-4136, (2017).

[11] Yousof, H. M., Altun, E., Ramires, T. G., Alizadeh, M., Rasekhi, M. "A new family of distributions with properties, regression models and applications", Journal of Statistics and Management Systems, 21(1): 163-188, (2018).

[12] Cordeiro, G. M., Ortega, E. M. \& da Cunha, D. C. C. "The exponentiated generalized class of distributions", Journal of Data Science, 11: 1-27, (2013).

[13] Cordeiro, G.M., Ortega, E.M.M., Nadarajah, S. "The kumaraswamy weibull distribution with application to failure data", Journal of the Franklin Institute, 347: 1317-1336, (2010). 
[14] Cordeiro, G.M., Hashimoto, E.M., Ortega, E.M.M. "The McDonald weibull model Statistics", Journal of Theoretical and Applied Statistics, 48: 256-278, (2014).

[15] Yousof, H. M., Affy, A. Z., Hamedani, G. G., Aryal, G., "The Burr X generator of distributions for lifetime data", Journal of Statistical Theory and Applications, 16: 288-305, (2017a)

[16] Yousof, H. M., Rasekhi, M., A_fy, A. Z., Alizadeh, M., Ghosh, I., Hamedani G. G. "The beta Weibull-G family of distributions: Theory, characterizations and applications", Pakistan Journal of Statistics, 33: 95-116, (2017b).

[17] Yousof, H. M., Alizadeh, M., Jahanshahiand, S. M. A., Ramires, T. G., Ghosh, I., Hamedani G. G. "The transmuted Topp-Leone G family of distributions: Theory, characterizations and applications", Journal of Data Science, 15: 723-740, (2017c).

[18] Oguntunde, P.E., "Generalisation of the inverse exponential distribution: statistical properties and applications", Phd. Thesis, Covenant University College of Science and Technology, Ota, Ogun State, 128-142, (2017a).

[19] Oguntunde, P., Khaleel, M. A., Ahmed, M. T., Adejumo, A. O. ,Odetunmibi O. A. "A new generalization of the lomax distribution with increasing, decreasing, and constant failure Rate",Hindawi Modelling and Simulation in Engineering, 1-7, (2017b). doi.org/10.1155/2017/6043169.

[20] Oguntunde, P. E., Odetunmibi, O. A., Adejumo, A. O. "On sum of exponentially distributed random variables", A Convolution Approach. European Journal of Statistics and Probability, 1(2): 1-8, (2013).

[21] Keller, A. Z., Kamath, A. R. R., \&Perera, U. D., “ Reliability analysis of CNC machine tools”, Reliability Engineering, 3(6): 449-473, (1982).

[22] Lin, C.T., Duran, B.S., Lewis, T.O., "Inverted gamma as a life distribution", Microelectronics Reliability, 29(4): 619-626, (1989).

[23] Abouammoh, A.M., Alshingiti, A.M. "Reliability estimation of generalized inverted exponential distribution", Journal of Statistical Computation and Simulation, 79(11): 1301-1315, (2009).

[24] Dey, S., Alzaatreh, A., Zhang, C., Kumar, D. "A new extension of generalized exponential distribution with application to ozone data", Ozone: Science and Engineering, 39(4): 273-285, (2017).

[25] Mahdavi, A., Kundu, D. "A new method for generating distributions with an application to exponential distribution", Communications in Statistics - Theory and Methods, 46(13): 6543-6557, (2017).

[26] Nadarajah, S., Okorie I. E. "On the moments of the alpha power transformed generalized exponential distribution", The Ozone: Science and Engineering, 1-6, (2017). doi/10.1080/01919512.2017.1419123

[27] Barreto-Souza, W., Simas, A. B. "Theexp-G family of probability distributions”, Brazilian Journal of Probability and Statistics, 27:84-109, (2013).

[28] Nadarajah, S., Nassiri, V., Mohammadpour, A. "Truncated-exponential skewsymmetric distributions", Statistics 48:872-895, (2014).

[29] Nassar, M., Alzaatreh, A., Mead, M., Abo-Kasem, O. "Alpha power Weibull distribution: properties and applications", Communications in Statistics -Theory and Methods 46:10236-10252, (2017). 
[30] Bourguignon M., Silva R. B., Cordeiro G. M. "The Weibull-G Family of probability distributions", Journal of Data Science, 12: 53-68, (2014).

[31] Unal, C., Cakmakyapan, S., Ozel, G. "Alpha power inverted exponential distribution: properties and application”, Gazi University Journal of Science, 31(3): 954-965, (2018).

[32] Smith, R. L., Naylor, J. C. "A comparison of Maximum Likelihood and bayesian estimators for the three-parameter Weibull distribution", Applied Statistics, 36: 258-369, (1987).

[33] Haq, M. A., Butt, N. S., Usman, R. M.,Fattah, A. A. “Transmuted power function distribution”. Gazi University Journal of Science, 29(1): 177-185, (2016).

[34] Merovci, F., Khaleel, M. A., Ibrahim, N. A., Shitan, M. "The beta type X distribution: properties with application”, Springer-Plus, (5): 697, (2016).

[35] Rastogi, M. K., Oguntunde, P. E. "Classical and Bayes estimation of reliability characteristics of the Kumaraswamy-Inverse exponential distribution", International Journal of System Assurance Engineering and Management, (2018). doi/10.1007/s13198-018-0744-7.

[36] Obubu, M., Oyamakin, S. O., Eghwerido J. T. "The gompertz length biased exponential distribution and its application to uncensored data", Current Trends on Biostatistics and Biometrics, 1: 52-57, (2019).

[37] Nichols, M. D., Padgett, W. J, "A bootstrap control chart for Weibull percentiles", Quality and Reliability Engineering International, 22: 141-151, (2016). 